\title{
Luminescent self-assembly formation on a gold surface observed by reversible 'off-on' switching of Eu(III) emission $\dagger$
}

\author{
Niamh S. Murray, ${ }^{a b}$ Suzi P. Jarvis ${ }^{b c}$ and Thorfinnur Gunnlaugsson ${ }^{* a}$ \\ Received (in Cambridge, UK) 19th May 2009, Accepted 6th July 2009 \\ First published as an Advance Article on the web 22nd July 2009 \\ DOI: $10.1039 / b 909938 g$
}

The formation of a self-assembly between a sensitising antenna and an Eu(III) functionalised cyclen complex 1.Eu, tethered to a gold surface via a $\mathbf{C}_{12}$ alkyl thiol spacer, is described where changes in the Eu(III) emission signal the formation, and dissociation, of a ternary complex.

The design and synthesis of functional material are of great current interest in chemistry and nanoscience. ${ }^{1-3}$ In particular, systems based on the use of self-assembly formation have been developed and studied in solution as mimics of macroscopic devices such as molecular switches and sensors, integrated logic gates, and as artificial molecular machines. ${ }^{4-8}$ Immobilising such systems onto various solid substrates offers an attractive means of forming practical devices that possess more highly ordered and populated species than would generally be present in solution. ${ }^{9,10}$ The use of luminescence to investigate the properties and function of supramolecular assemblies has been extensively employed due to its non-invasive nature, fast response time and high sensitivity. ${ }^{11-13} \mathrm{We}^{14,15}$ and others ${ }^{16}$ have developed several examples of functional lanthanide supramolecular devices and investigated their luminescence properties in solution ${ }^{17}$ and soft materials, ${ }^{18}$ and also more recently on nanoparticles. ${ }^{19,20}$ While the unique photophysical properties of the lanthanides make them ideal for observing self-assembly formation and for sensing, to the best of our knowledge, their use on gold substrates has not been achieved to date. Such modification would further increase their potential use in sensing applications, for instance, by combining them with atomic force microscope (AFM)-based micromechanical sensors, ${ }^{21}$ which could result in the development of dual functioning sensory systems. We have previously demonstrated the formation of luminescent ternary complexes in solution formed through self-assembly using sensitising antennae and coordinatively unsaturated $\mathrm{Eu}(\mathrm{III})$ or $\mathrm{Tb}$ (III) complexes. ${ }^{22}$ Herein, we demonstrate that by anchoring such a lanthanide complex, 1.Eu, onto a gold substrate, via an alkyl thiol linker, the lanthanide luminescence is 'switched on' upon formation of a self-assembly between 1.Eu and a sensitising antenna, 2. The emission of this assembly can then be 'switched off' upon addition of $\mathbf{3}$, an anion possessing an antenna with

${ }^{a}$ School of Chemistry, Centre for Synthesis and Chemical Biology, Trinity College Dublin, Dublin 2, Ireland.E-mail: gunnlaut@tcd.ie; Fax: + 3531671 2826; Tel: + 35318963459

${ }^{b}$ Centre for Research on Adaptive Nanostructures and Nanodevices (CRANN), Trinity College Dublin, Dublin 2, Ireland

${ }^{c}$ School of Physics, University College Dublin, Belfield, Dublin 4, Ireland

$\dagger$ Electronic supplementary information (ESI) available: Supplementary figures. See DOI:10.1039/b909938g

unfavourable excited state energies for the population of the Eu(III) excited state.
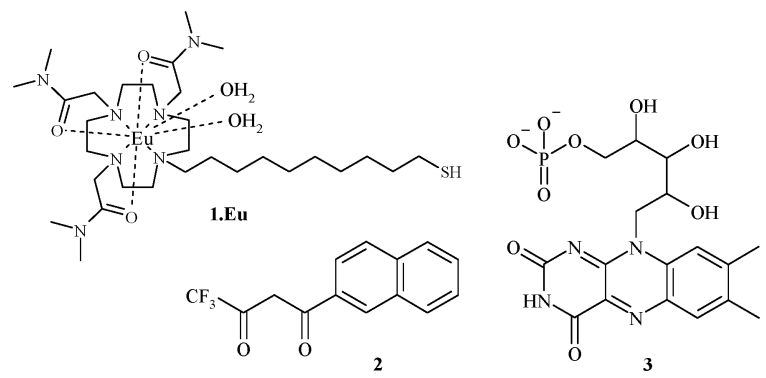

The rationale for our design is shown in Fig. 1. The gold substrates employed were made by firstly sonicating discs (9 $\mathrm{mm}$ diameter) of mica in ethanol, and then evaporating a thin layer of gold (approximately $10 \mathrm{~nm}$ ). Following evaporation, the samples were immersed in $1 \mathrm{mM}$ solutions of 1.Eu and left overnight. This was followed by thoroughly rinsing the surface with ethanol and drying under $\mathrm{N}_{2} . \ddagger$ Similarly, samples possessing 1.Eu and 2 (1-Eu.2) and 1.Eu and the phosphate anion, flavin monophosphate 3 (1-Eu.3), were also made for comparison purposes. The FTIR analysis of these modified gold surfaces showed the presence of both 1.Eu and 1.Eu.2 with a number of characteristic resonances being identified (see ESI $\dagger$ ). For instance, the $\mathrm{C}=\mathrm{O}$ stretch assigned to the carboxy amide of $\mathbf{1} \cdot \mathbf{E u}$ appeared at $1624 \mathrm{~cm}^{-1}$ with a shoulder at $1636 \mathrm{~cm}^{-1}$, while the asymmetric and the symmetric $\mathrm{CH}_{2}$ stretches of the alkyl thiol chain appeared at 2930 and $2856 \mathrm{~cm}^{-1}$, respectively. The addition of the antenna, which gave 1.Eu.2, showed an additional new band appearing at $1657 \mathrm{~cm}^{-1}$, assigned to the $\mathrm{Eu}(\mathrm{III})$ coordinated antenna (see ESI $\dagger$ ). The surface structures of these modified gold samples in water were also imaged using AFM in contact

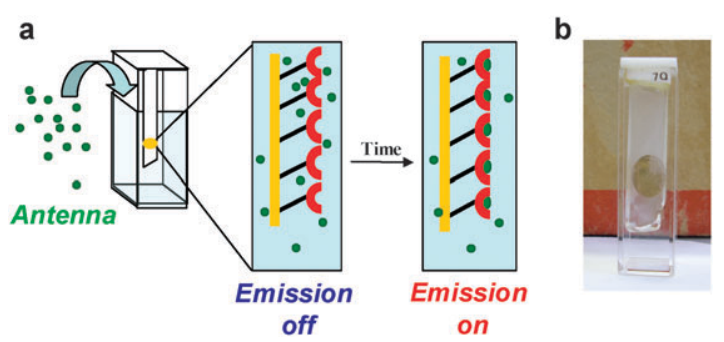

Fig. 1 (a) Schematic representation of a gold surface (on mica) functionalised with a self-assembled monolayer of 1.Eu mounted on a quartz slide. The Eu(III) emission is switched 'off' prior to the addition of the antenna $\mathbf{2}$, which forms a ternary complex with $\mathbf{1} \cdot \mathbf{E u}$ and the emission is switched 'on'. (b) Image of the gold sample, functionalised with 1·Eu. 
mode, using silicon nitride cantilevers. The image of the unmodified gold surface (see ESI $\dagger$ ) showed defined islands of gold, possessing grain sizes of approximately $100 \mathrm{~nm}$, and upon functionalisation with 1.Eu.2, the morphology of the surface was significantly changed.

It has previously been reported that gold quenches lanthanide luminescence. ${ }^{23}$ However, as such quenching is distance dependent, we proposed that our design would minimise any such quenching due to the presence of the $\mathrm{C}_{12}$ spacer between 1.Eu and the surface. To establish this, the delayed emission of 1.Eu and 1.Eu.2 and 1.Eu.3 was recorded upon excitation at $281 \mathrm{~nm}$. The results are shown in Fig. 2 and clearly demonstrate that, for 1.Eu alone, no lanthanide emission is observed. This is not surprising as 1.Eu does not possess a sensitising antenna, such as $\mathbf{2}$, necessary for the successful population of the Eu(III) excited state ${ }^{5} D_{0}$. Upon excitation of the antenna in 1.Eu.2, the presence of an intense $\mathrm{Eu}(\mathrm{III})$ emission was clearly visible. This demonstrated the successful sensitisation of the ${ }^{5} \mathrm{D}_{0}$ state, with the appearance of line-like emission bands occurring at 595,616, 650 and $700 \mathrm{~nm}$, respectively, due to the deactivation of ${ }^{5} \mathrm{D}_{0} \rightarrow{ }^{7} \mathrm{~F}_{J}(J=1,2,3$ and 4). Of these, the hypersensitive $\Delta J=2$, which is highly sensitive to the local coordination environment of the metal ion, was the most intense. This confirms the formation of a ternary self-assembly complex by the direct coordination of $\mathbf{2}$ to the Eu(III) ion.

To further investigate this sensitisation process from $\mathbf{2}$, we varied the excitation wavelength (see ESI $\dagger$ ), which showed that the expected $\mathrm{Eu}(\mathrm{III})$ centred emission was observed, but also a band corresponding to a second order scattering effect (see inset, Fig. 2).

To confirm that the observed emission was indeed due to selfassembly formation, we used 1.Eu.3, which only gave rise to minor $\mathrm{Eu}(\mathrm{III})$ emission, centred at $616 \mathrm{~nm}$, upon excitation at $281 \mathrm{~nm}$. This result confirms that previously seen in solution, that the antenna 3 fulfils the coordination requirements of $\mathrm{Eu}(\mathrm{III})$, but lacks the ability to efficiently populate the lanthanide excited state.

We next monitored the evolution of the Eu(III) emission upon formation of a self-assembly between 1.Eu and 2. This was done using two experimental methods: (a) by immersing a 1.Eu modified gold sample into a solution of $\mathbf{2}$, and recording the evolution of the $\mathrm{Eu}(\mathrm{III})$ emission as a function of time, and (b) by carrying out a "titration" by treating a 1.Eu modified gold sample with a solution of $2\left(1 \times 10^{-4} \mathrm{M}\right)$ for 1 minute,

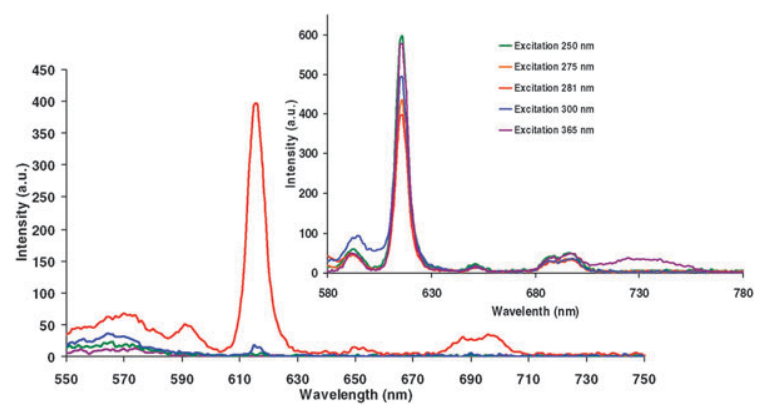

Fig. 2 The phosphorescent emission spectra observed from the gold sample in the absence (purple base line) of $\mathbf{1} \cdot \mathbf{E u}$, and of the modified gold surface of 1.Eu (green), 1.Eu.2 (red) and 1.Eu.3 (blue) upon excitation at $\lambda_{\text {excitation }}=281 \mathrm{~nm}$. Inset: The emission of $\mathbf{1} \cdot \mathbf{E u} \cdot \mathbf{2}$ upon $\lambda_{\text {excitation }}$ at $250,275,281,300$, and $365 \mathrm{~nm}$, respectively.

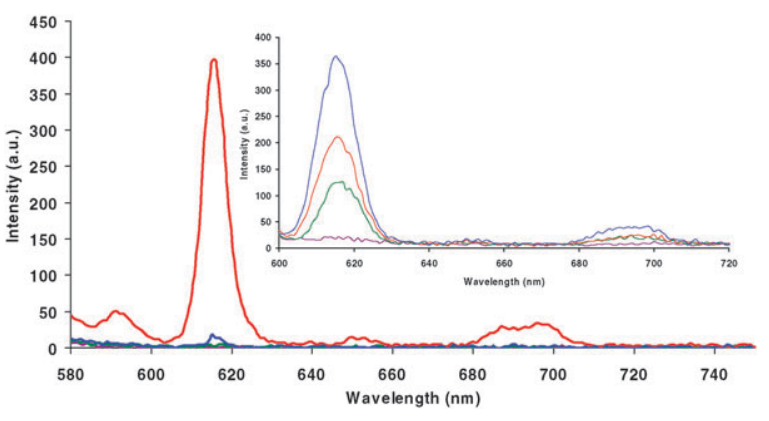

Fig. 3 The $E u(I I I)$ emission arising from 1.Eu functionalised gold surface, upon excitation at $281 \mathrm{~nm}$, before (blue) and after immersion for 1 minute in a solution of $2\left(1 \times 10^{-4} \mathrm{M}\right)$ (green) and after immersion overnight (red). Inset: The changes observed after 1 minute (green), 10 minutes (red), and one hour (blue) exposure of gold 1.Eu sample to a solution of $2\left(1 \times 10^{-4} \mathrm{M}\right)$ upon excitation at $281 \mathrm{~nm}$.

followed by rinsing with ethanol and drying under $\mathrm{N}_{2}$ prior to recording the $\mathrm{Eu}(\mathrm{III})$ emission. This process was then repeated a number of times on the same sample. The results for method (a) are shown in Fig. 3, and show that the emission observed for the $\Delta J=2$ to 4 manifolds is "switched on", demonstrating the formation of a ternary complex between 1.Eu and $\mathbf{2}$. Also shown in Fig. 3 are the changes observed within the first hour of forming this self-assembly, which reveals that the emission is fully switched on within one hour. This process was fully reproducible. Moreover, the emission intensity arising from a sample of 1.Eu stored in a solution of $\mathbf{2}$ remained the same over a period of days. The Eu(III) emission was also clearly 'switched on' using method (b) (see ESI $\dagger$ ). Unfortunately, we were unable to accurately determine the excited state decay of the $\mathrm{Eu}(\mathrm{III}){ }^{5} \mathrm{D}_{0}$ state of these gold samples, which had fully decayed within $0.5 \mathrm{~ms}$ (see $\mathrm{ESI} \dagger$ ), and hence we were unable to determine the hydration state of $\mathbf{1} \cdot \mathbf{E u}$ before and after the addition of $\mathbf{2}$.

Having established the formation of the 1.Eu.2 ternary complex on the gold surface, we further evaluated the stability of the 1.Eu.2 gold sample by measuring the Eu(III) emission arising from 1.Eu.2 after storing the gold sample in ethanol for 24 hours (see $\mathrm{ESI} \dagger$ ), in the absence of $\mathbf{2}$. Unlike that seen above, the results here showed that the $\Delta J=2$ transition of the Eu(III) emission intensity was reduced by almost $85 \%$ from that shown in Fig. 3. In an attempt to investigate this loss of $\mathrm{Eu}(\mathrm{III})$ emission, we re-immersed the sample in a solution of $\mathbf{2}$ and recorded the $\mathrm{Eu}(\mathrm{III})$ emission, which was restated to almost the same intensity as shown before. This suggests that the self-assembled monolayer of 1.Eu.2 on the gold surface potentially undergoes a reorganisation in the absence of $\mathbf{2}$, which may facilitate a more efficient quenching of the Eu(III) emission by the gold surface. By dehydrating the sample in air, the emission was also significantly quenched (see ESI $\dagger$ ), but could be 'switched back on' upon hydration in a solution of $\mathbf{2}$, which also supports this theory. However, we are currently investigating the issue further.

As was shown in Fig. 2, and previously discussed above, no significant $\mathrm{Eu}(\mathrm{III})$ emission was observed from gold samples functionalised with 1.Eu.3 upon excitation at $281 \mathrm{~nm}$ (or at the $\lambda_{\max }$ of the antenna 3 ). Consequently, we next evaluated if the $\mathrm{Eu}(\mathrm{III})$ emission observed from 1.Eu.2 in Fig. 3 could be 


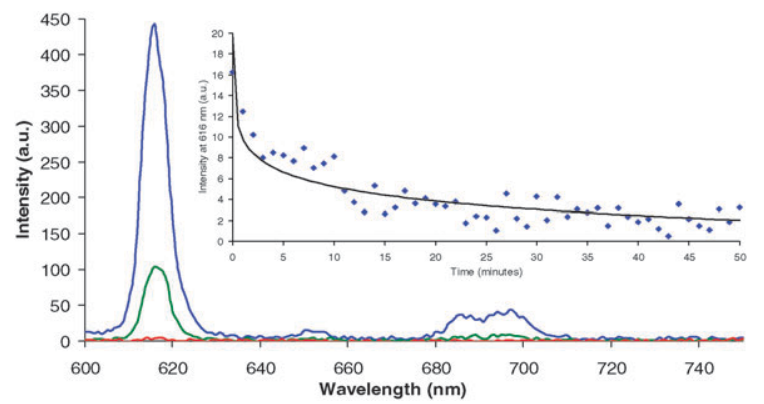

Fig. 4 The $\mathrm{Eu}(\mathrm{III})$ emission arising from 1-Eu functionalised gold surface, upon excitation at $281 \mathrm{~nm}$, before (blue) and after 1 minute immersion in a solution of $3\left(1 \times 10^{-4} \mathrm{M}\right)$ (green) and after immersion overnight (red). Inset: the changes in the Eu(III) emission of observed from 1.Eu.2 in the presence of $\mathbf{3}$ over 50 minutes, upon recording the changes every minute.

quenched by immersing the 1.Eu.2 gold sample into a solution of 3. The changes observed in the Eu(III) emission in this competitive media for the first 50 minutes are shown in Fig. 4, and clearly show that the Eu(III) emission is rapidly quenched, even after immersion for only a few minutes. These results complement those seen in Fig. 2, and in our previous work, and demonstrate that $\mathbf{2}$ is displaced from $\mathbf{1} \cdot \mathbf{E u} \cdot \mathbf{2}$ by $\mathbf{3}$, with the concomitant formation of $\mathbf{1} \cdot \mathbf{E u} \cdot \mathbf{3}$, which is non-luminescent. This is also clear from the kinetic experiment shown as an inset in Fig. 4. Hence, these results demonstrate that the Eu(III) emission from 1.Eu on a gold surface can be "switched on" by an external 'input' (e.g. 2), and that this process can be reversed upon addition of a second chemical 'input' (e.g. 3).

In summary, we have demonstrated that monothiol conjugated cyclen complexes, possessing a coordinatively unsaturated Eu(III) centre, can be used to develop a luminescent lanthanide-based selfassembled monolayer on a gold surface. We show that the Eu(III) emission from this gold sample is "switched on" in the presence of an appropriate sensitising antennae, such as $\mathbf{2}$. Furthermore, this emission can then be "switched off" upon addition of a competitive coordinating ligand $\mathbf{3}$. We are in the process of further exploring the properties of 1.Eu. $\mathbf{2}$ and related lanthanide complex modified gold surfaces for sensing applications.

We thank Science Foundation Ireland (SFI), CRANN (for postgraduate studentship to NSM) and TCD for financial support. We would also like to thank Dr Julien Massue for his initial help with the synthesis of 1-Eu and Dr Susan J. Quinn for helpful discussions and advice during the course of this project.

\section{Notes and references}

$\ddagger$ Immobilization on a substrate allows for a much higher concentration of 1.Eu than would normally be used in solution. A rough estimate of the number of 1.Eu on a $9 \mathrm{~mm}$ disc (given that thiol bond has a footprint of $22 \AA^{2}$, and that approximate available surface area is $63 \mathrm{~mm}^{2}=63 \times 10^{9} \AA^{2}$ ) allows for the incorporation of $\sim 2.8 \times 10^{9}$ 1.Eu complex on the gold surface used in Fig. 1, a number vastly in excess of what is used in solution measurements.

1 V. Serreli, C. F. Lee, E. R. Kay and D. A. Leigh, Nature, 2007, 445 , 523; E. R. Kay, D. A. Leigh and F. Zerbetto, Angew. Chem., Int. Ed., 2007, 46, 72; C. D. Meyer, C. S. Joiner and J. F. Stoddart, Chem. Soc. Rev., 2007, 36, 1705.

2 P. Ghosh, G. Han, M. De, C. K. Kim and V. M. Rotello, $A d v$. Drug Delivery Rev., 2008, 60, 1307; N. L. Rosi and C. A. Mirkin, Chem. Rev., 2005, 105, 1547.
3 C. M. G. dos Santos, A. J. Harte, S. J. Quinn and T. Gunnlaugsson, Coord. Chem. Rev., 2008, 252, 2512; T. Gunnlaugsson, M. Glynn, G. M. Tocci (née Hussey), P. E. Kruger and F. M. Pfeffer, Coord. Chem. Rev., 2006, 250, 3094; E. B. Veale and T. Gunnlaugsson, J. Org. Chem., 2008, 73, 8073; H. He, M. A. Mortellaro, M. J. P. Leiner, S. T. Young, R. J. Fraatz and J. K. Tusa, Anal. Chem., 2003, 75, 549.

4 V. Balzani, A. Credi and M. Venturi, Molecular Devices and Machines, Wiley-VCH, Weinheim, 2nd edn, 2008.

5 C. S. Bonnet and T. Gunnlaugsson, New J. Chem., 2009, 33, 1025; A. P. de Silva and S. Uchiyama, Nat. Nanotechnol., 2007, 2, 399; A. P. de Silva, S. Uchiyama, T. P. Vance and B. Wannalerse, Coord. Chem. Rev., 2007, 251, 1623.

6 U. Pischel, Angew. Chem., Int. Ed., 2007, 46, 4026; A. P. de Silva, Y. Leydet, C. Lincheneau and N. D. McClenaghan, J. Phys.: Condens. Matter, 2006, 18, S1847.

7 J. F. Stoddart and H. M. Colquhoun, Tetrahedron, 2008, 64, 8231.

8 G. J. Brown, A. P. de Silva, M. R. James, B. O. F. McKinney, D. A. Pears and S. M. Weir, Tetrahedron, 2008, 64, 8301.

9 K. Ai, B. Zhang and L. Lu, Angew. Chem., Int. Ed., 2009, 48, 304.

10 J. Shen, L.-D. Sun and C.-H. Yan, Dalton Trans., 2008, 5687; J. D. E. T. Wilton-Ely, Dalton Trans., 2008, 25; M.-C. Daniel and D. Astruc, Chem. Rev., 2005, 104, 293.

11 J.-C. G. Bunzli and C. Piguet, Chem. Soc. Rev., 2005, 35, 1048; J.-C. G. Bunzli and C. Piguet, Chem. Rev., 2002, 102, 1897.

12 T. L. Borgne, J. M. Benech, S. Floquet, G. Bernardinelli, C. Aliprandini, P. Bettens and C. Piguet, Dalton Trans., 2003, 20, 3856.

13 N. Kerbellec, L. Catala, C. Daiguebonne, Al. Gloter, O. Stephan, J.-C. G. Bünzli, O. Guillou and T. Mallah, New J. Chem., 2008, 584; J. P. Leonard, P. Jensen, T. McCabe, J. E. O'Brien, R. D. Peacock, P. E. Kruger and T. Gunnlaugsson, J. Am. Chem. Soc., 2007, 129, 10986.

14 J. P. Leonard, C. B. Nolan, F. Stomeo and T. Gunnlaugsson, Top. Curr. Chem., 2007, 281, 1; T. Gunnlaugsson and F. Stomeo, Org. Biomol. Chem., 2007, 5, 1999; T. Gunnlaugsson and J. P. Leonard, Chem. Commun., 2005, 3114; J. P. Leonard and T. Gunnlaugsson, J. Fluoresc., 2005, 15, 585.

15 C. M. G. dos Santos and T. Gunnlaugsson, Dalton Trans., 2009, 4712; A. M. Nonat, A. J. Harte, K. Sénéchal-David, J. P. Leonard and T. Gunnlaugsson, Dalton Trans., 2009, 4703; S. E. Plush and T. Gunnlaugsson, Dalton Trans., 2008, 3801; C. S. Bonnet, M. Devocelle and T. Gunnlaugsson, Chem. Commun., 2008, 4552; T. Gunnlaugsson and J. P. Leonard, Dalton Trans., 2005, 3204.

16 M. Andrews, R. H. Laye, L. P. Harding and S. J. A. Pope, Polyhedron, 2008, 27, 2365; J. J. Yu, D. Parker, R. Pal, R. A. Poole and M. J. Cann, J. Am. Chem. Soc., 2006, 128, 2294; J.-C. G. Bünzli, Acc. Chem. Res., 2006, 39, 53; D. Parker, Chem. Soc. Rev., 2004, 33, 156; S. Faulkner and S. J. A. Pope, J. Am. Chem. Soc., 2003, 125, 10526.

17 S. E. Plush and T. Gunnlaugsson, Org. Lett., 2007, 9, 1919; J. P. Leonard, C. M. G. dos Santos, S. E. Plush, T. McCabe and T. Gunnlaugsson, Chem. Commun., 2007, 129; C. M. G. dos Santos, P. B. Fernandez, S. E. Plush, J. P. Leonard and T. Gunnlaugsson, Chem. Commun., 2007, 3389; K. SénéchalDavid, J. P. Leonard, S. E. Plush and T. Gunnlaugsson, Org. Lett., 2006, 8, 2727; K. Sénéchal-David, S. J. A. Pope, S. Quinn, S. Faulkner and T. Gunnlaugsson, Inorg. Chem., 2006, 45, 10040; A. J. Harte, P. Jensen, S. E. Plush, P. E. Kruger and T. Gunnlaugsson, Inorg. Chem., 2006, 45, 9465.

18 C. P. McCoy, F. Stomeo, S. E. Plush and T. Gunnlaugsson, Chem. Mater., 2006, 18, 4336; T. Gunnlaugsson, C. P. McCoy and F. Stomeo, Tetrahedron Lett., 2004, 45, 8403.

19 J. Massue, S. E. Quinn and T. Gunnlaugsson, J. Am. Chem. Soc., 2008, 130, 6900.

20 C. S. Bonnet, J. Massue, S. E. Quinn and T. Gunnlaugsson, Org. Biomol. Chem., 2009, 7, 3074.

21 S. Kim and K. D. Kihm, Appl. Phys. Lett., 2006, 89, 061918; N. McLoughlin, S. L. Lee and G. Hahner, Appl. Phys. Lett., 2006, 89, 184106; J. Fritz, M. K. Baller, H. P. Lang, H. Rothuizen, P. Vettiger, E. Meyer, H.-J. Güntherodt, C. Gerber and J. K. Gimzewski, Science, 2000, 288, 316.

22 T. Gunnlaugsson, A. Harte, J. P. Leonard and M. Nieuwenhuyzen, Supramol. Chem., 2003, 15, 505; T. Gunnlaugsson, A. Harte, J. P. Leonard and M. Nieuwenhuyzen, Chem. Commun., 2002, 2134. 23 H. Kuhn, J. Chem. Phys., 1970, 53, 101. 